is met with in the central portion of thick bars of Swedish iron, kept for some weeks at a temperature below their melting point, but high enough to give rise to recrystallisation. We then get a complete separation of free iron from a compound containing some carbon, and a crystalline structure which, as far as mere form is concerned, most closely corresponds with that of meteoric iron, as may be at once seen on comparing them.

These facts clearly indicate that the Widmanstatt's figuring is the result of such a complete separation of the constituents and perfect crystallisation as can occur only when the process takes place slowly and gradually. They appear to me to show that meteoric iron was kept for a long time at a heat just below the point of fusion, and that we should be by no means justified in concluding that it was not previously melted. Similar principles are applicable in the case of the iron masses found in Disco, and it by no means follows that they are meteoric because they show the Widmanstatt's figuring. Difference in the rate of cooling would serve very well to explain the difference in the structure of some meteoric iron, which do not differ in chemical composition; but, as far as the general structure is concerned, I think that we are quite at liberty to conclude that all may have been melted, if this will better explain other phenomena. On this supposition we may account for the separation of the iron from the stony meteorites, since under conditions which brought into play only a moderate gravitative force, the melted iron would subside through the melted stone, as happens in our furnaces; whilst at the same time, as shown in my paper read at the meeting of the British Association in 1864, where the separating force of gravitation was small, they might remain mixed together, as in the Pallas iron, and others of that type.

In conclusion I would say that though from want of adequate material for investigation $I$ feel that what $I$ have so far done is very incomplete, yet I think that the facts I have described will, at all events, serve to prove that the method of study employed cannot fail to yield most valuable results, and to throw much light on many problems of great interest and importance in several different branches of science.

\section{MENDELEEF'S RESEARCHES ON MARIOTTE'S LAW}

FROM researches on the depression of the mercury results the possibility of introducing a precise correction relative to the volume of gas contained between the surface of the mercury and the horizontal plane which touches the summit of the meniscus, In all my researches $I$ introduce each time a cor* rection relative to this volume.

The volume of the reservoir which contains the mercury and the gas under various pressures undergoes two kinds of variations; first, those which are due to the difference between the pressures which act on the two sides of the vessel, and second, those which depend on differences in the volume of mercury. The compressibility of the reservoirs employed in the researches has been always determined by experiment, and their change of volume produced by the introduction of mercury can be de* termined by surrounding the vessel filled with mercury by another filled with the same material. When the height in the two vessels is the same, the capacity of the vessel is that which exists at the time of equality of pressure on the external and internal surfaces of the vessel. If we empty a part of the external vessel the capacity of the vessel changes in the same manner as when we fill or when we empty the vessel. Experiments of this kind have shown the possibility of determining the changes of capacity depending on the quantity of mercury. The relative corrections have in each case been introduced into the calculations.

All the practical side of the subject-the desiccation of the gas, the complete abstraction of the remains of the gas from the apparatus, the hermetical junction of the parts of the apparatus by means of mastic and mercury stop-valves, the means of main-

2 Consinued from p. 45\%. taining the gases and the mercury at a constant temperature, the calibration of the tubes, and a number of other details have had to be elaborated more or less anew. All this will be found described in my work "On the Elasticity of Gases." I. have published this work only in Russian, not having means suff cient to publish a translation of a work so voluminous, and desiring to conform to the custom existing among savants of al countries of describing their labours in their mother-tongue, in order to present to the scientific literature of the country where they live and work a gift in proportion to their powers.

My desire was to investigate the subject in its minutest details in order to eliminate every possibility of doubt as to the causes which determine the deviations observed from the Boyle. Mariotte Law. $I$ know that that law is firmly established, and I believe it will remain so. Not less great is the certainty in the mind that rarefied gases approach the perfect state. That certainty I had also on commencing my experiments. It was necessary then to desermine as completely as possible all the circumstances on which depend the facts contrary to the opinion generally held. This is why I have modifed the apparatus, improved the methods, and employed in this work more than three years without interruption. Now so far as regards low pressures the work is finished, and I have obtained definitely certain proofs of the rigorous accuracy of my first observations.

The experiments which I have made with Kirpitchoff have proved that not only for air, but also for hydrogen, and even for carbonic acid, the deviations are posiive when the gas is sub jected to o very small pressure; it is fond, moreover, that these deviations increase in yroportion to the variation from the normal pressure. The same thing has been found in a new seris of experiments undertaken by me with $M$. Hemillan. The experiments are described in tome ii. of my work on the "Elasticity of Gases," which I have just publishea. A brief extract on this subject is published in the Ann. de Chimie et de Physique, October, 1876 . I shall quote only the results obtained by us from the experiments made in 1875 and in the begining of 1876.

Into a new apparatus we have introduced several further inprovements, of which the chief are:-(I) The baromanometer the metre, and the reservoir, containing the gas and the mercury, have been placed in the same bath full of water; (2) We have succeeded in producing a complete vacuum in the barometric chamber; (3) The bath was maintained at an almost uniform temperature by means of an agitator, and the small differences in the temperatures of the varous layers have been determined by a differeatial thermometer; (4) The junction between the air reservoir and the baromanometer has been made, not ouly without the aid of a tap, but also without the use of mastic. ${ }^{1}$ Thus the gas was surrounded only by the glass and the mercury. We shall confine ourselves to a summary of the results of our experiments, made between 650 and 20 millimetres' pressure, with four gases - $\mathrm{H}$, air, $\mathrm{CO}^{2}$, and $\mathrm{SO}^{\circ}$

I. If, starting with a cextain small pressure, we arrive at pressures smaller still, we find for all gases positive deviations, viz., $\frac{d(p v)}{d p}>0$; the gases, then, are in this case less compressed ap than Mariotte's Law requires. Similar deviations were also observed for hydrogen by M. Regnaait between $\mathrm{I}$ and $z^{\circ}$ an mo spheres, and M. Natterer for all gases between Ioo and 3,000 atmospheres.

2. Under small pressures and for all gases, the value of the positive deviations, i.e., the numerical quantity (or magnitude) $\frac{d(p v)}{d p}$, increases when the initial pressure diminishes. Thus, for example, for bydrogen at 400 millimetres-

$$
\begin{aligned}
& \frac{d(p v)}{d p}=+0.000002 \\
& \text { and at r2o millimetres } \\
& \frac{d(p v)}{d p}=+0.000010
\end{aligned}
$$

3. For gases like $\mathrm{CO}^{2}$ and $\mathrm{SO}^{2}$ we find near the amospheric pressure, negative deviations; e.g., "for $\mathrm{CO}^{2}, p_{0}=635, p_{1}=200$, $p_{0} v_{0}=10,000, p_{1} v_{1}=10,029$; but, under less pressures still, the deviations become positive even for $\mathrm{CO}^{2}$ and $\mathrm{SO}^{2}$. For example, for $\mathrm{CO}^{2}, p_{0}=190, p_{1}=64, p_{2}=22, p_{0} v_{0}=10,000, p_{1} v_{1}=$ $9,996, p_{2} z_{2}=9,983 ;$ for $\mathrm{SO}^{2}, p_{0}=190, p_{1}=60, p_{2}=22$, $p_{0} z^{0}=10,000, p_{1} v_{1}=10,010, p_{2} v_{2}=9,996$.

4. The existence of positive and negative deviations for the

$x$ To attain this end the gas-vessel and the branch of the baromanometer are soldered together by a capillary tube made of a single piece. 
same gas, observed by means of the same apparatus, according to the amount of pressure, and the conformi:y in the various series of experiments, prove that the results obtained do not depend on any constant errors in the methods employed, but that they are really caused by the nature and the essential qualities of the gases investigated.

5. The variations from Mariotte's Law under very weals pressures being very small, it is necessary, in determining them, to make the reading of the pressures, the volumes, and the temperatures (absolute $t=273^{\circ}$ ) with a precision of two-thous andths of these total values; thus, e.g., if $p_{0}=0.200 \mathrm{~m} ., p_{1}=0.100 \mathrm{~m}$., and $v_{0}=2,500$ gr., $z_{1}^{\prime}=5,000 \mathrm{gr}$. of mercury $(t=20)$, it will be necessary to determine the pressures with a precision of $0.01 \mathrm{~mm}$., the volumes to $0.1 \mathrm{gr}$. of mercury, and the temperatures to $0^{\circ}$ I 1 of a degree.

The results will be doubt?ul if the precision is less. ${ }^{1}$ Thus it is found that under a certain small pressure gases presen: positive deviations from Mariotte's Law; even gases like sulphurous acid and carbonic acid, which under high pressures show consider. able negative deviations. It is the same with air. M. Regnault commenced his researches with pressures which exceeded that of the atmosphere, and obtained negative deviations.

In $1874 \mathrm{I}$ effected with all the care possible the determination of the deviations for air under pressures of from 650 to 2,000 milimetres, and towards the end of 1875 and in the beginning of 1876 , in a special apparatus provided with compound manometers, I repeated the same experiments with $M$. Bogusski for pressures from 700 to 3,000 millimetres with air, hydrogen, and carbonic acid. These researches proved the rigorous accuracy of M. Regnault's conclusions. Air and carbonic acid were found to be subject under these pressures to negative deviations, greater for carbonic acid than for air; and hydrogen, for these sane presures, was found to present positive deviations. At present we are continuing the same kind of experiments for pressures of more than three metres.

Thus hydrogen, under all pressures, commencing with zero and ending with a pressure infinitely great, presents throughout posilive deviations; at no pressure does it follow Boyle's Law rigorously, and it never presents negative deviations. Increased pressures always give a greater volume than what might be expected according to the variation of the pressures. Air under pressures less than 600 millimetres also presents positive devi. ations; but under pressures greater than that of the atmosphere its deviations become negative, and under pressures which exceed IOO atmospheres its compressibility again becomes positive. Consequently for this gas there are two pressures at which it follows Boyle's Law ; the one is very nearly that of the atmo: sphere, the other lies between 30 and roo atmospheres. These pressures, under which the changes of the sign of compressibility occur, will be different for carbonic acid; viz., under pressures less than that of the atmosphere the change of sign is found at nearly 200 millimetres, and for higher pressures it commences near that which corresponds to 70 metres of mercury, if we base our researches on this point on the observations of $\mathrm{Dr}$. Andrews on the compressibility of carbonic acid gas for temperatures above $31^{\circ}$. For lower temperatures this point will probably correspond to the passage of carbonic acid into the liquid state. Consequently wilh a change of temperature the pressure at which the change of sign of compressibility occurs, changes also. For sulphurous acid the sign of compressibility under pressures lower than that of the atmosphere changes at about forty millimetres of pressure. But even this gas, so easily liquefiable, under low pressures, has always a positive compressibility. There is not then, and there cannot be, a gas which is iigorously subject to Mariotte's Law under small pressures.

The idea of an absolute gas belongs, then, to the number of fictions which find no confirmation in facts. We cannot, then, suppose that with the decrease of density or with the increase of the vis viva of gaseous nolecules, gases approach a state in which they follow Boyle's Law. Then (the density diminishing, the velocity of the molecules increasing, that is to say, the pressure diminishing, the temperature increasing, and the molecular weight diminishing) they all tend towards another state characterised by the expression $\frac{d(p v)}{d p}>0$; i.e., they are assimilated to solid and liquid bodies, when the condensation reaches its limit. We

I It is by these causes that the want of conformity in the experiments of Siljeström is sufficiently explained ( $P$ ogg. Annt., Afril and May, 1874 see also the Bull. de l"Acad. de Sc. de St. Pétersboutg, t. xix, p. 466, and Berichte der deatschen chem. Gesell., t. viii., P. I, 339; t. viii., Pp. 576 and 749), and of M. Amagat (Comptes Rendus, April $17, \times 876$.) must believe that there is a limit of condensation and a lim t of 1arefaction. If we take, in fact, a mass of non-volatile liquid, and if we submit it to pressures infinitely great and infinitely small, we shall see it change volume; but in the two cases, we shall have finite volumes, capable of measurement, and even differing little for one and the same body. It is the same with gases, if we admit that for pressures anproaching zero, gases contract according to the same law as that which we can deduce from our compression experiments under pressures less than that of the atmosphere, or as hydrogen contracts. Under great pressures, or under pressures excessively small, every gas resembles a solid or liquid body, and possesses two limits of compressibility. The volumes which correspond to these limits are very different, but there is always reason for believing that they exist.

Without launching into hypotheses to explain these limit volumes (such, e.g., as the supposition that molecules in themselves possess volume), I will confine myself to the question of the matter of celestial space. What is the luminous ether? One of two things-either an elastic independent matter, sai gfneris, or the gas of the atmuspheres of celestial bodies, considerably rarefied. In the latter case it is necessary to admit the absence of limits in the atmospheres and a condensation of the ether greater and greater in proportion as we approach a celestial body (sun or planet). There are many arguments for and againt both hypotheses. On the one hand, spectrum analysi: leads us to conclude that the material of all heavenly bodies is identical ; on the other hand, it proves the diversity of the composition of atmospheres. This is why we abstain from solving the question in its essence. But spectrum analysis does not speak less in favour of the former hypothesis, because it shows the diversity of composition of our terrestrial atmosphere from that of many of the other celestial bodies. In the researches on the resistance of celestial matter to the movernent of the planets, there appears also to be a confirmation of the former of these suro hypotheses, for neither planets nor comets show any diminution in the excentricity of their crbits, which would be an inevitable consequence of motion in a rarefied medium, as has been observed in the case of Enclie's comet. Exact investigations on the movement of that comet, repeated in recent times by M. von Asten, the Pulkowa astronomer, show clearly the advances towards the sun at perihelion, although in the beginning $\mathrm{M}$. von Asten had nut noticed them. But that comet at perihelion was found only at one-third of the distance which separa e: the sun from the earth, i.e., it was nearer to the sun than Mercury. It is possible that it passed near to the limits of the solar atmosphere. Faye's comet, as is lnown, does not present these same diversities, but its perihelic distance is about $\mathbf{1} 68$, that of Encke's comet being only about $0^{\circ} 33$; it exceeds it then so much that their comparison would only serve to confirm the hypothesis of a solar atmosphere. If we admit a linit for the atmospheres, we must expect in gases, for small pressures, exactly that zind of variation from Boyle's Law which I observed in rarefied gases.

To prove that gases under very small pressures, as well as under very considerable pressures, vary from the Boyle-Marriotte Law is by no means the same as to deny the truth of that law; I feel that I ought to state this most explicitly. For a long time the law of gravitation could not be made to accord with the perturbations latterly these perturbations have proved the best crinfirmation of the laws of gravitation. In the present case it may be the same. There are three laws for gases: that of Boyle and Marriotte, $p v=$ const. ; that of Gay Lussac, $v_{t}=v_{0}(\mathbf{1}+a t)$; and that of Ampère and Gerland $\frac{a v}{m}=$ const. ( $a$ being the molecular weight, and $n$ the mass). Their ensemble is expressed for all gases in general by the equation-

$$
a p v=845(273+t) m
$$

where $a$ is the atomic weight $(H=\mathbf{r}), p$ the pressure in kilo. grams per square metre, $v$ the volume in cubic metres, $m$ the weight in kilograms, $t$ the centigrade temperature. 'This is, however, only a first approximation. In the second member of the equation there must be additional terms which express a function of $p$ and of $a$, very small for the ordinary mean values of $p$, and which become of a sensible magnitude only when $p$ is very small or very great. To find this function is a question of the future, and demands the labours of a great number of investigators. My aim is to be able to furnish some experimental data which will permit of judging of the form of that function. This work requires many new processes, measuring apparatus of a high 
degree of precision, many varied arrangements as well as experienced assistants. That idea has secured me the protection of His Imperial Highness the Grand Duke Constantine and the support of the Imperial Russian Technical Society.

I thus conclude this communication which $I$ have the honour to send you. The researches on the co-efficients of dilatation of gases in their general features confirm the accuracy of the deductions drawn from the observations on compressibility. But that matter is in process of elaboration, and only a part of the researches has been completed. Consequently $I$ abstain from expounding all the details of the subject. I shall only remark that the true coefficient of dilatation of air under constant pres. sure and with variable volume is found to be greater for pressures near to almospheric pressure than the number generally accepted, notably from the researches which I have made with M. Kaiander, it is equal to $0^{\prime} 003683$ if we take $100^{\circ} \mathrm{C}$. as the temperature of boiling water under an atmospheric pressure of $760 \mathrm{~mm}$. in latifude $45^{\circ}$.

If you do not find devoid of interest more ample details on this subject, as also on the determination of the weight of a litre of air, I shall have the greatest pleasure in explaining to the English public through your interesting journal the essential points of the researches made on this subject in my laboratory.

St. Petersburg, January $\mathrm{r}$ DE MENDELEEF

\section{OUR ASTRONOMICAL COLUMN}

Binaxy Stars.-From one of the very careful and complete investigations on the orbits of the revolving double stars, by which Dr. Doberck is so greatly contributing to our knowledge of the motions of these interesting objects, we lave an orbit of $\xi$ Scorpii which is probably a very near approximation to the true one.

We have called the star by what appears to be its correct designation, $\xi$ Scorpii, but few stars have been subjected to more varied nomenclature than the one in question. In Dr. Doberck's paper, published in No. 2,12I of the Astronomische Nachrichten, in all probability through one of the typographical errors which have of late so much disfigured this periodical, the star is styled $\zeta$ Libræ. It has been previously very commonly lettered $\xi$ Libræ, and it is 51 Libræ of Flamsteed; Secchi, in Astron. Nach., No, $\mathrm{r}, 614$, calls it $\rho^{\prime} \mathrm{Libra}$, though we are ignorant upon what precedent.

Dr. Doberck's elements are those of a very nearly circular orbit, and it may be remembered that some thirty years since Mädler gave elements for circular motion with a period of revolution of about 104 years. In Astron. Nach, No. 1,683, Dr. Thiele gave the results of a very complete discussion of the measures up to 1856 , in which he has assumed that Sir W. Herschel's angle on the night of his discovery of the duplicity of the star has been registered correctly, though a doubt has been entertained upon this point. He thus arrives at a highly excentrical elliptic orbit with a period of a little over forty-nine years. It should be remarked that from the near approach of the mag. nitudes of the components forming the double star $\xi$ Scorpii (it is more correctly a triple star), an error of $180^{\circ}$ in the measured angle of position is by no means an improbable one.

Dr. Doberck assumes that Sir W. Herschel's angle of 1782 requires this correction, and deduces an orbit in which the period of revolution is nearly twice that of Thiele, and which therefore approaches the period originally assigned by Mädler. His elements are as follow :-

Peri-astron passage 1859.62 .

\begin{tabular}{|c|c|c|c|c|c|}
\hline Node & $15^{\prime}$ & Inc & ation & & $68^{\circ} 42^{\prime}$ \\
\hline Node to peri-astr & on on & orbit & $\ldots$ & & $89^{\circ} 16^{\prime}$ \\
\hline Eccentricity & $\ldots$ & $\cdots$ & & $\ldots$ & 0.0768 \\
\hline Semi-axis major & $\cdots$ & $\cdots$ & $\ldots$ & $\ldots$ & $\mathrm{I}^{\prime \prime} \cdot 26$ \\
\hline ution & ... & . & $\ldots$ & & yo years. \\
\hline
\end{tabular}

A full comparison with the measures up to the present time, appears in the Astronomische Nachrichten.

In the same number Dr. Doberck gives first elements of that exceedingly difficult object $\gamma$ Coronse Borealis, in which the period of reyolution is $95 \frac{1}{2}$ years, and the peri-astron passage $1843 \%$. The distance calculated from this orbit is still under two-tenths of a second, but it will increase, until towards the end of the first decade of the next century the components, according to Dr. Doberck's calculation, will be separated by more than $\mathrm{o}^{\prime \prime} \cdot 8$.

The Annular Eclipse of the Sun, 1737, March 1.Prof. Grant, in his "History of Physical Astronomy," mentions this eclipse as the first annular one of which we have any detailed account. This phase passed over Edinburgh, where it was ob. served by Maclaurin, the well-known mathematician, by Short, the optician, Lord Aberdour, and others. The times were determined by Maclaurin by a pendulum clock of Grabam's, and he was also furnished with a meridian instrument by the same maker, with the aid of which the clock was rated by Short for " a long time before and after the eclipse." The clock used by Lord Aberdour, who was located in Edinburgh Castle, was compared with Maclaurin's at noon on the day of the eclipse, and in addition signals were exchanged between the Castle and Maclaurin's station at the college. Both observers determined the duration of the annular phase to have been $5 \mathrm{~m}$. $48 \mathrm{~s}$.

The following elements of this eclipse have been deduced from a similar system of computation as regards the moon's place to that adopted for other eclipses to which reference has been made from time to time in this column, a system which gives results for the total solar eclipse of $17 \times 5$ agreeing very closely with the observations of Flamsteed and Halley.

G.M.T. of conjunction in R.A., March I, at 3 h. Im. $3^{\text {Is. }}$

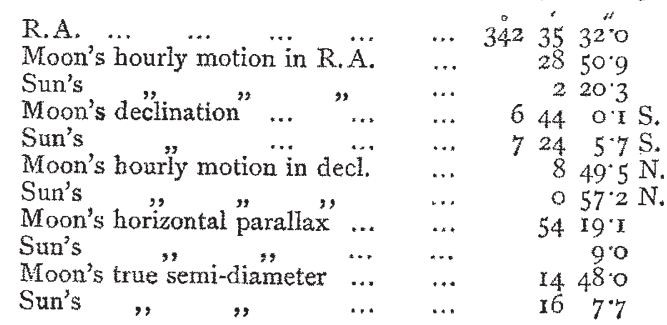

The equation of time was I $2 \mathrm{~m}$. 4OS. subtractive from mean time. The following were points upon the central line by the above elements :-

Long. Io ro W. Lat. $5453 \mathrm{~N}$. " $726 \mathrm{~W} ., \quad 5542 \mathrm{~N} . \mid$, o $4 \mathrm{~W} ., 25736 \mathrm{~N}$.

If reduction equations are founded upon a direct calculation for Edinburgh, there result for any place not far distant :-

$\cos w=27^{*} 63^{6} 69-[\mathrm{I} 596 \mathrm{~m}] \sin l+\left[\mathrm{x}^{*} 220 \mathrm{r}^{8}\right] \cos l, \cos \left(L-5^{x^{\alpha}} \mathrm{x}^{r_{4}} 4\right)$

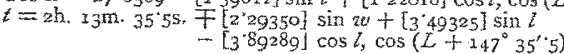

where $l$ is the geocentric latitude of the place, $L$ its longitude from Greenwich + if $E_{,}$- if $W$, and $t$ is expressed in Green. wich mean time; the upper sign is to be used for beginning of annular phase, and the lower for the ending. The quantities within square brackets are logarithms.

The calculated duration of annularity at Edinburgh is

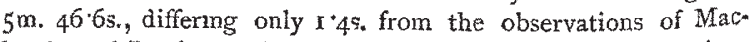
laurin and Lord Aberdour, but the middle of this phase is given later by Im. 5 Is. At other places mentioned in Maclaurin's memoir on this eclipse, published in the Philosophical Transactions, the duration of the annulus was as follows:-At Alnwick, $2 \mathrm{~m}$. Os. ; at Crosby, near Ayr, 5m. 54s.; at Montrose, 6m. 27s. ; and at St. Andrew's, 6m. I2s. At Aberdeen, which was very near the central line, the annulus was formed at $3^{\mathrm{h} .} 43^{\mathrm{m}}$. os. local mean time, and continued $6 \mathrm{~m}$. 30s. On the east coast of Scotland, where the duration of annulus was longest, it did not exceed $6 \mathrm{~m} .35$. The eclipse is not given annular at Morpeth, therein agreeing with the observation. 